\section{Wie geht es weiter?}

Wenn die Umsystematisierung abgeschlossen ist, das Wallstädt-Programm zur Verfügung steht und letzte offene Fragen geklärt sind, werden voraussichtlich ab Anfang 2017 mithilfe studentischer Hilfskräfte Fach für Fach die Daten im Lokalsystem aDIS geändert, die Bücher mit neuen Signaturschildern versehen und in der neuen Ordnung wieder ins Regal gestellt. Für die Datenänderungen im Verbundkatalog ist eine automatische Einspielung aus den Listen des „Programms Oberfell“ angestrebt.

Über all dies berichten wir dann in einem späteren Beitrag.

Katja Selmikeit

\title{
RVK-Umsystematisierung leicht gemacht!
}

Signaturbildung mit dem "Programm Oberfell"

Die Umstellung der Lesesäle auf die Regensburger Verbundklassifikation (RVK) bedeutet auch einen Umstieg auf deutlich längere und komplexere Signaturen. Zur Erleichterung der Umsystematisierung entwickelte Jörg Oberfell, stellvertretender Leiter der IT-Abteilung der WLB, eine eigene Software. Sie unterstützt bei der Signaturbildung, das heißt bei der Vergabe der Notation, eines oder mehrerer Cutter sowie eventueller weiterer Signaturbestandteile wie etwa einer Bandzählung. Um eine Menge von Titeln im „Programm Oberfell" bearbeiten zu können, wird zunächst eine entsprechende Suche in der SWB-Verbunddatenbank (WinIBW) durchgeführt - zum Beispiel nach allen Titeln mit einer Hauptlesesaal-Signatur. Die gefundenen Datensätze werden in eine Datei exportiert und von dort in das Programm importiert, wobei alle relevanten Kategorien in ein internes Format überführt werden. Die Datei erhält die Endung .rvk. Sie kann bei Bedarf in mehrere Dateien geteilt werden, zum Beispiel nach Signaturenbereichen zur Bearbeitung durch unterschiedliche FachreferentInnen.

Öffnet man die Datei im „Programm Oberfell”, so erscheinen die Titel bzw. Bände in einer Kurztitelliste (obere Bildschirmhälfte). Wählt man einen davon aus, so bekommt man hierzu die ausführlichen bibliografischen Daten angezeigt (unten links). Im RVK-Bearbeitungsbereich findet schließlich die Bildung der neuen Signatur statt (unten rechts). Der erste Signaturbestandteil ist die RVK-Notation.

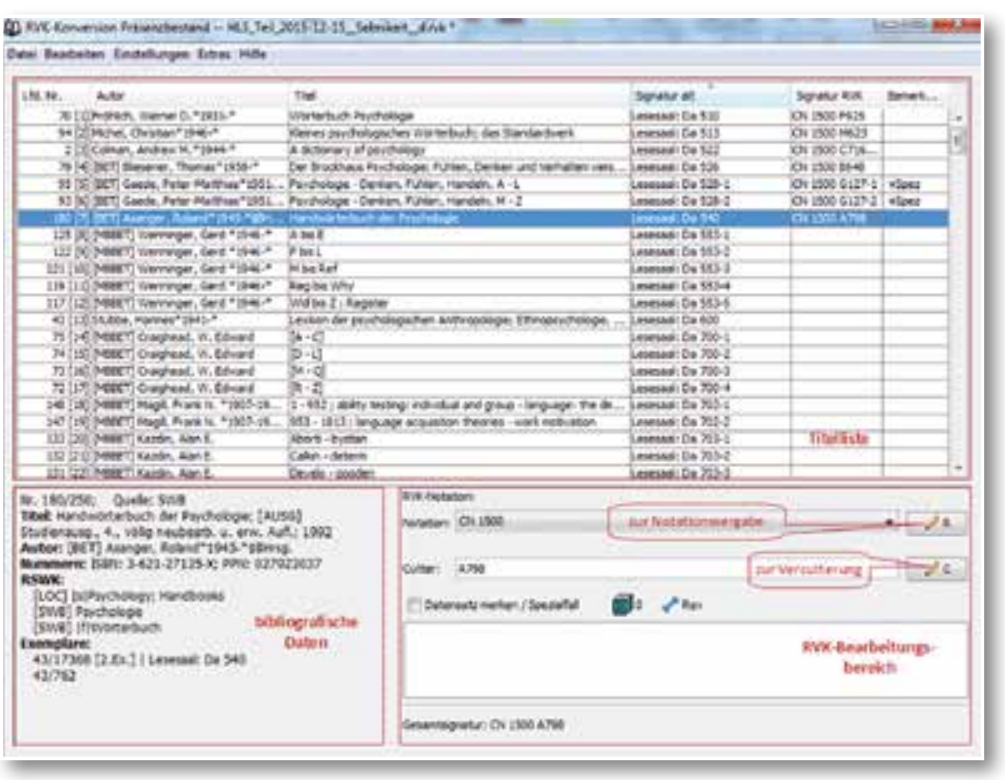

Abb. 1: Hauptfenster mit Titelliste und Bearbeitungsfunktionen

Sind für den betreffenden Titel bereits eine oder mehrere Notationen aus dem SWB importiert worden, so kann die gewünschte Notation direkt aus der Drop-Down-Liste im Bearbeitungsbereich ausgewählt werden. Anderenfalls wird über einen Button das Dialogfenster zur Notationsvergabe geöffnet.

Die Drop-Down-Liste aus dem Bearbeitungsfenster erscheint hier erneut. Steht keine Notation aus dem SWB zur Verfügung oder möchte man eine alternative Notation vergeben, so kann man diese - in der Regel nach Recherche in der RVK Online frei eintragen. Zwei weitere Optionen sind für noch zu beantragende bzw. bereits beantragte neue RVK-Notationen vorgesehen. 


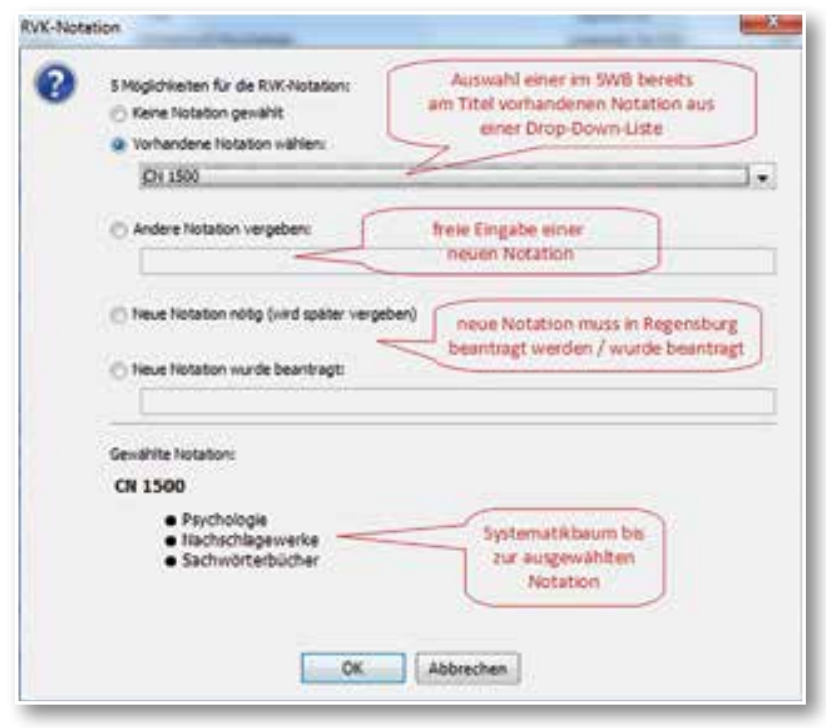

Abb. 2: Dialogfenster zur Vergabe einer Notation

Bei Auswahl oder Eintrag einer gültigen Notation bekommt man im unteren Bereich deren Benennung sowie alle übergeordneten Hierarchieebenen als Systematikbaum angezeigt. Diese Information zieht das Programm aus der hinterlegten XMLDatei der RVK.

Als zweiter Signaturbestandteil folgt der Cutter (Cutter-Sanborn-Notation oder CSN). Im entsprechenden Bearbeitungsfenster wählt man das Wort, aus dem dieser gebildet werden soll (in der Regel der Nachname einer beteiligten Person oder ein Wort aus dem Titel) aus einer Drop-Down-Liste aus; ist das gewünschte Wort nicht enthalten oder möchte man ein anderes vercuttern, so kann man es frei eingeben.

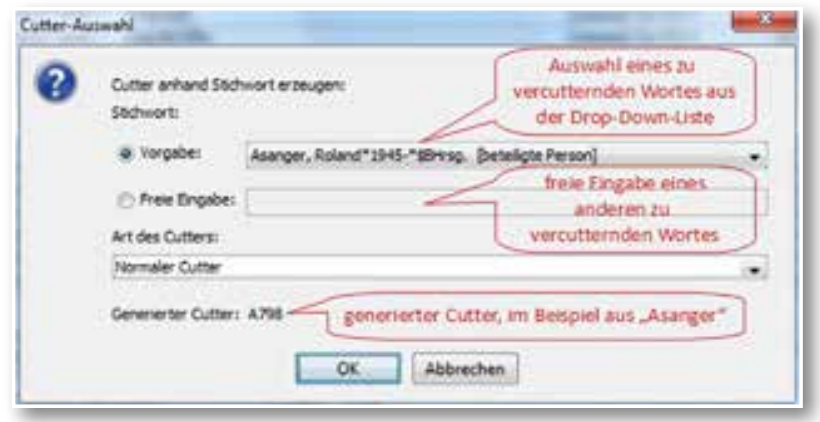

Abb. 3: Dialogfenster zur Erzeugung eines Cutters

Den daraus automatisch generierten Cutter schließt das Programm mit einem Leerzeichen an die Notation an. Eventuelle weitere Cutter werden auf dem gleichen Weg generiert und können anschließend bei Bedarf auf die gewünschte Zeichenzahl gekürzt werden.

Zurück im Hauptbearbeitungsfenster lassen sich eventuelle weitere Signaturbestandteile wie zum
Beispiel die Bandzählung frei in das Cutter-Feld eintragen. Ist zu einer Titelaufnahme mehr als ein Band vorhanden, können in einem weiteren Dialogfenster mit wenigen Schritten alle zugehörigen Bände erfasst werden. Dies erleichtert die spätere Bearbeitung durch Erzeugung von Barcodes und Signaturetiketten für jeden einzelnen Band.

Da während der Bearbeitung einer Liste laufend neue Titel in den Lesesaal gelangen, die vorerst noch nach der alten Lesesaalsignatur aufgestellt werden, muss sie kurz vor Beginn der physischen Umstellung und Datenänderung der betreffenden Titel noch einmal aktualisiert werden. Nicht mehr im Lesesaal stehende Bände (die etwa ins Magazin umgestellt wurden) werden dabei aus den Listen entfernt und neue hinzugefügt, die dann noch umsystematisiert werden müssen.

Ist eine Liste fertig bearbeitet, so werden aus dem Programm heraus Laufzettel zum Einlegen in die umzustellenden Bücher gedruckt. Je zwei Barcodes gewährleisten ein einfaches und tippfehlerfreies Arbeiten bei der Datenänderung: Mithilfe einer Lesepistole kann ein Titel über die als Barcode verschlüsselte PPN aufgerufen werden, und über einen zweiten Barcode wird die RVK-Signatur im Lokalsystem bzw. für den Signaturetikettendruck in den - ebenfalls eigens im Haus programmierten Etiketteneditor eingelesen.

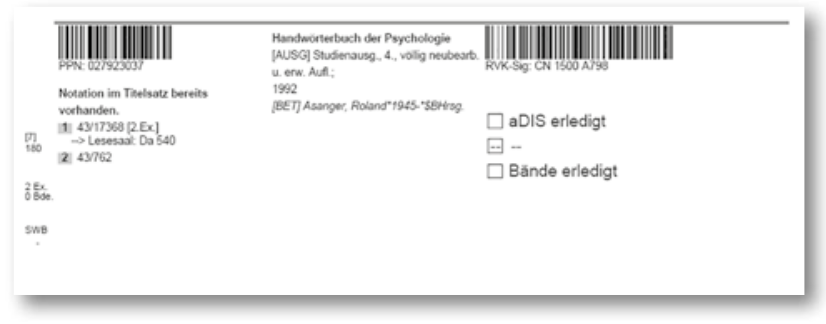

Abb. 4: Laufzettel mit Barcodes

Bei Bedarf geben wir das Programm gern an interessierte Bibliotheken weiter, können jedoch über eine grundlegende Einführung und Installationshilfe hinaus leider keinen Support leisten.

Katja Selmikeit / Jörg Oberfell 